\title{
Diagnosing ship propulsion by vibration measurement for navigation safety
}

\author{
A. Charchalis \\ Gdynia Maritime University, Mechanical Faculty, Poland
}

\begin{abstract}
Presently, as a result of the technical progress, more and more complicated machines are being used in our everyday life. This is especially the case in relation to technology used at sea, where highly specialised services are needed. Sophisticated marine devices require special diagnostic methods that take into account the specific conditions of use of this type of machinery. In this paper we present the diagnostic systems elaborated to support the exploitation of the vessel power plant with gas turbines. During engine assembly, the rotating components are mounted with great attention in order to minimise shaft unbalance. However, even while applying the highest standards, factors such as machines' imperfection or differential thermal expansion cause a small residual unbalance of the gas turbine rotor. The dynamic problems of Marine Gas Turbine Engines are directly related to such basic elements as rotors, bearings, struts of bearings, engine body and the type of substructure.
\end{abstract}

Keywords: diagnosing, vibroacoustics, gas turbine engines, ship propulsion plant, vessel propulsion system.

\section{Introduction}

The propulsion systems of warship vessels and especially combat vessels are constructions where enormous power is applied. The power of the installed propulsion system reaches $100 \mathrm{MW}$. In order to achieve the required high power production gas turbine engines are used. The contemporary gas turbines used on warships can achieve power up to $30 \mathrm{MW}$, at the same time possessing little unit mass (even up to $0,2 \mathrm{~kg} / \mathrm{W}$ ). 
The specific conditions at sea in which the marine gas turbine engines are used influence to a considerable degree their exploitation requirements and may cause their increased wear or even lead to a break-down.

Although marine conditions influence all types of engines, their impact on the gas turbines is notorious [1]. This is due to:

- the great volume intensity of air flow through the engine, up to $80 \mathrm{~m}^{3} / \mathrm{s}$,

- the high level of the load on the engine,

- the complexity of a thorough inspection of the engine and in some cases incapacity to perform a full examination,

- the requirement of the high level of purity of fuel, oil and air,

- work mostly on partial loads.

The engines on the vessels are operated in conditions of permanent waving and great stroke loads. These kinds of loads put a considerable strain on the elements of the engine, especially on the complex system of seals and bearings. The air that passes through the engine introduces sea-water and salt residues, oil vapours, sucked exhausts of the working engines and, in the coastal regions, industrial dusts.

\section{Basic diagnostic system for naval gas turbine engines}

For the above reasons, professional technical supervision of a high standard is required for the proper use of naval gas turbines. Such requirements can hardly be fulfilled by the crews of small vessels. Therefore, it was decided to support the crews of such vessels by introducing the "Base Diagnostic System for Naval Gas Turbine Engines" (BDS) [2].

The system should be applied for the periodical inspection of engine conditions, particularly with respect to:

- annual maintenance,

- the necessity of the prolongation of mean time between major repairs,

- identification of abnormal running found during routine maintenance.

The Base Diagnostic System consists of a series of diagnostic positions and provides the possibility of complex examination of engine conditions by EDP (Electronic Data Processing) application. The BDS is capable of determining the prognosis for the future operation of the engine. An operating decision is obtained on the basis of appropriately prepared measurements of the engine parameters. They are subsequently converted into diagnostic parameters according to the elaborated flow diagrams for computer programs.

From a technical point of view, the BDS is equipped with a special supervising-measuring device capable of carrying out numerous (foregoing) tasks pursuant to the measured values of various parameters of the engine.

In order to perform all tasks securing the proper gas turbine operation the system is equipped with the following special devices:

- computer measuring system of start-up and lay-off parameters, and of operational parameters for measurements of vibration parameters and their analysis, 
- position for oil examinations on metal particle contents and other impurities,

- programmable analyser of high-changeable signals,

- endoscopes,

- $\quad$ automatic test equipment for safety devices and supervising-measuring apparatus of the engines,

- computer database.

The methods of gathering diagnostic parameters for the condition evaluation of different sub-assemblies of the engine are presented in Figure 1.

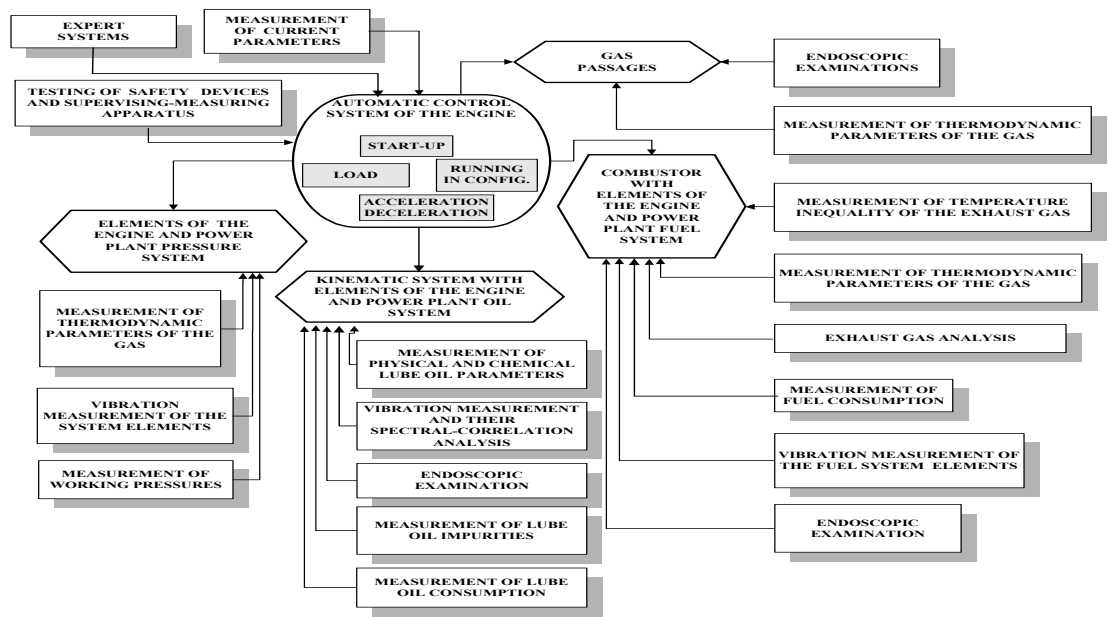

Figure 1: $\quad$ Methods and means of gathering diagnostic parameters.

In order to obtain reliable data on diagnostic parameters, investigations of the gas turbines installed in the presented propulsion system were carried out by means of the multi-symptom diagnostic model. One of the main features of this model is recording and analysing vibroacoustic signals [3]. The investigations were aimed at determining permissible in-service unbalance and appropriate assemblage of turbine rotors on the basis of selected vibroacoustic parameters, and - finally - determining their permissible operation time resources. The analysis was based on the following assumption: if technical state degradation of gas turbine rotor sets is a function of their operation time (at a load spectrum assumed constant) then it is possible to select from the recorded vibration signal spectrum such parameters whose changes can be unambiguously assigned to the operation time $[4,5]$. Shaft misalignment between engines and reduction boxes and the propeller and reduction box constitutes a second important issue. Dynamic reactions, which result from exceeding the allowable alignment deviations of the torque transmission elements, may cause failures of the propulsion system and even lead to loss of movability of the vessel within a relatively short time. Therefore, diagnostic control of the gas turbine power plant in operation becomes strictly necessary. 
Appropriate assembling of the main engines and other torque transmission elements (inclusive of propellers) is determined by a set of tolerated dimension and geometrical location requirements, referred to as geometrical dimension assembling chain. Both typical and modular power plants are prone to coaxiality deviation from its permissible values and in consequence may be subject to possible failures of one or more elements of the propulsion system. Excessive deviation can lead to loads on bearings and gear teeth that considerably exceed the calculated values and lead to their premature failures.

\section{Theoretical assumption of rotor dynamics}

The application of a computer simulation for diagnosing the technical condition of gas turbine rotor sets should be taken into consideration within the process of the calculation of parameters and elaboration of the model. In fact, this takes place in practice. The problem arises when the manufacturer does not include this kind of know-how in the technical specification addressed to the user. Such a situation may occur, for instance, in the case of navy vessels equipped with gas turbine engines that are intended for export. During engine assembly, the rotating components are mounted with great care with the main objective of minimising shaft unbalance. However, even with the highest standard of attention, such factors as machine imperfections or differential thermal expansion cause small residual unbalances of the gas turbine rotor. The dynamic problems of Marine Gas Turbine Engines (MGTE) are directly related to such basic elements as rotors, bearings, struts of bearings, engine body, type of substructure as well as to hydro- and meteorological conditions during sea trials and gas flown parameters inside the engine. The quality of operation and stability of MGTE also depend on such parameters. Dissipation of energy in rotating machines displays as torque, revolutions, temperature, gas flown and vibration. Vibrations are connected to rotors unbalancing, oversize of tolerated axis slope of shafts, abrade of blade tips with the inner roller, wear of axis and radial bearings, asymmetry of springiness and damping characteristics of rotors and their parts and irregular gas flown forces. The emission of vibration provides a lot of information including the diagnosis on the technical state [5]. Measurements of vibration, their identification, classification and mathematical analysis, including trend function, provide information on the present technical state of the engine and allow prediction of the wear process of the engine in the future.

It is worthwhile pointing out that every rigid body has six degrees of freedom, however a deformated body has unlimited degrees of freedom. Rotating machines like MGTE have an amount of degrees of freedom equal to the sum of all degrees of freedom of the engines' parts reduced by the amount of rigid nodes connecting these elements of the engine. Each part of the engine can be represented by physical characteristics obtained from vibration measurements or from modelling of geometry and material - a rigidly joined structure. The application of a specified model of rigid body gives ordinary differential equations. The deformated body needs partitive differential equations. The second assumption is considerably more complicated but it can achieve a 
closeness to the real object, especially when it works in a wide range of rotary speeds. For these reasons, the second model was chosen as the more appropriate method. The scheme of diagnostics model MGTE is presented on figure 2 .

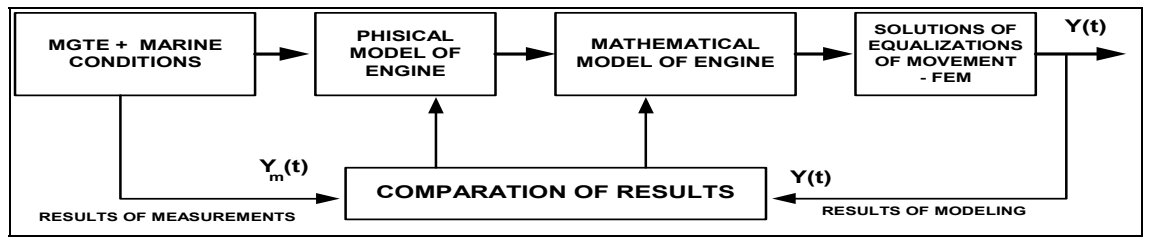

Figure 2: $\quad$ Scheme of the diagnostics model.

The residual unbalance occurs on each and all stages of the rotor but it may be represented by two vectors of unbalance at both ends of shaft. They have different values and phase shifts. This FE individual and average model creates responses of unbalancing and it can be used for comparison with vibration reports resulting from the measurements. The most sensitive unbalance response point at the GT engine is the front frame over a vertical strut. This is due to the effect of minimal thermal expansion for radial and axis vibration in this point. The model is linear so it is evident that the response is directly proportional to the amount of unbalance [4]. It should be noted however that the real engine response is unlikely to be linear over a wide range. Furthermore, the result obtained can be accepted only as a statistical approximation of the dynamic engine response.

The construction of the rotor is subject to forces originating from different sources. These forces not only result from the unbalancing or gas-flown forces but also from the vibration of the vessel's hull, enclosure module, propeller, shaft and gearbox. The list of potential sources is extensive. Generally, these are axis slopes, shaft cracks, blade tips, cracks or wanes. The focus has been mainly directed at these sources during modelling and investigation on real objects. Loss of material affects the changes of moments of inertia of rotated parts. They cause the displacement of the main axis of inertia, which is not coincident to the axis of rotation. Finally, they constitute the main sources of unbalancing and vibration of the rotor.

The main purpose of this research was to identify the sensitive vibration symptoms represented by the residual unbalancing of rotors and forces from the misalignment of shafts. FEA (Finite Element Analysis) is used successfully for a wide range of problems and may also be used for the modelling and analysis of a rotor system. Presently, the diagnostics teams commonly use FEA and rotordynamics in conjunction with vibration analysis for the detection and identification of unbalancing and misalignment of shafts. A linear model obeys the basic principle of linear superposition. Once applied to a structure, this means that displacement resulting from a combination of structural loads is the sum of the displacements due to each individual load making up the combination. Unfortunately, not enough structural information was available to create a FEA model for marine gas turbine engines. This is typical when the 
product of operation has been manufactured in a foreign country. So, the decision was taken to apply a passive method of investigation in order to determine reliable symptoms of unbalancing and misalignment by using statistical methods and verifying them by endoscopic examination [5]. For initial analysis from the first to fourth harmonics of amplitude of the velocity of vibration, the dimensionless parameters S1 and S2 were taken as sensitive symptoms.

\section{Diagnosis of rotors unbalancing}

The quality of operation and stability of MGTE are equally connected with the state of such parameters. Dissipation of energy in rotating machines displays as torque, revolutions, temperature, gas flown and vibration. Vibrations are connected to:

- rotors unbalancing;

- $\quad$ oversize of tolerated axis slope of shafts - misalignment;

- blade tips with the inner roller;

- wear of axis and radial bearings;

- asymmetry of springiness and damping characteristics of rotors and their parts;

- irregular gas flown forces.

The emission of vibration provides a lot of information including opinion on the technical state of the device. The measurements of vibration, their identification, classification and mathematical analysis, including trends, provide information on the present condition and allow for predicting wear process in the future. Vibration analysis of MGTE during sea trials is accomplished by two different procedures: on-line - in real time, and off-line - periodic or single measurements.

To perform the analysis the following measurement instruments were used: the FFT-2148 analyser and PULSE v 9.0 software of Bruel and Kjaer, enabling the collection and processing of measured data. Measuring transducers (accelerometers) were fixed to steel cantilevers located on the flange of the low-pressure (LP) compressor only. It was decided to carry out the investigations with the use of the transducer fixed to the LP compressor flange due to the lack of transducers and equipment suitable for measuring signals at temperatures as high as $200^{\circ} \div 300^{\circ} \mathrm{C}$ occurring on the high-pressure (HP) compressor flange.

The fixing accelerometers' cantilevers are characterised by a vibration natural resonance frequency value, and differ enough from harmonic frequencies, due to the rotation speed of the turbine rotors and their harmonics. The measurements were taken perpendicularly to the rotation axis of the rotors over the main bearings. This choice was made on the basis of the theoretical consideration of excitations due to unbalanced shaft rotation, and the results of preliminary investigations of the object [6]. With respect to signals, usable for the "defectsymptom" relation, the following magnitudes were selected by the turbines' producer: 
- Y $-1^{\text {st }}$ harmonic RMS value of vibration velocity amplitude connected with the LP and HP rotor of compressor;

- Yrms - RMS value of vibration velocity amplitude within the range of $35 \mathrm{~Hz} \div$ $400 \mathrm{~Hz}$.

The choice was justified by the time-between-repair values scheduled by the turbines' producer. For the purpose of these investigations a simplification was made that consisted of assuming values of the after-repair turbine vibration symptoms as equal to those of a new turbine. The turbines' producer specified the following limit values of RMS vibration velocity amplitude: Yrms $=24$ $\mathrm{mm} / \mathrm{s}, \mathrm{Y}=17 \mathrm{~mm} / \mathrm{s}$.

In order to obtain uniform diagnostic procedures regarding unbalance assessment of the turbine rotors the dimensionless parameters characterising such states were applied. On the basis of theoretical considerations as well as a result of other diagnostic investigations carried out for some years the following parameters were selected as the most sensitive, [6]:

$\mathrm{S} 1$ - ratio of the mean vibration velocity amplitude of a given rotor $\left(1^{\text {st }}\right.$ harmonic) and the velocity component relevant to the $2^{\text {nd }}$ harmonic excitation frequency of the rotor in question; $\mathrm{S} 1>1$

$\mathrm{S} 2$ - ratio of the mean vibration velocity amplitude of a given rotor $\left(1^{\text {st }}\right.$ harmonic) and the velocity component relevant to the $3^{\text {rd }}$ harmonic excitation frequency of the rotor in question; $\mathrm{S} 2>1,5$.

One of the most important elements of the off-line system is the database. For the purposes of further consideration, idle load and full power were selected. Each spectrum was transferred as matrixes $M_{S}$ and copied like fingerprints to the database. Spectra were not synchronized to the revolutions of rotors, as at the same loads in different air temperature conditions they differ from each other. As a result, the following preparation for the procedure of identification has been established. This appeared to be an important point of analysis because of the sensitivity of typical spectra - as shown on Figure 3.

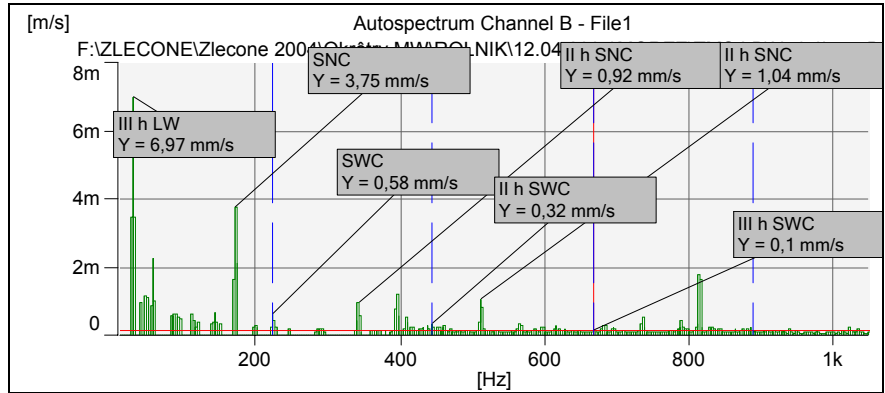

Figure 3: $\quad$ Typical pattern spectra DR 77 engines.

\section{Diagnosis of shafts misalignment}

The usual measurement methods of coaxiality parameters of the propulsion system require disassembling the protection covers of shafting between the 
engines and reduction gears. Measurement requirements cause a suspension of the COGAG-system operation for about $8-10$ days and it is of course an intrusive method. The vibroacoustic method presented in this paper allows assessment of the permissible values of the alignment parameters without stopping the exploitation use of the vessel. Moreover, the presented results are intended to form the database for elaboration of an on-line monitoring system of coaxial of torque transmission elements, applicable to the COGAG propulsion system in question.

Dynamic reactions, resulting from exceeding allowable alignment deviations of the torque transmission elements may cause failures of the propulsion system and even lead to loss of movability of the vessel within a relatively short time [2]. Therefore, diagnostic control of the gas turbine power plant in operation becomes necessary.

Appropriate assembling of the main engines and other torque transmission elements (inclusive of propellers) is determined by a set of tolerated dimension and geometrical location requirements, referred to as geometrical dimension assembling chain [3]. Both typical and modular power plants are prone to coaxiality deviation from its permissible values and in consequence to possible failures of one or more elements of the propulsion system. The excessive deviation can lead to excessive loads on bearings and gear teeth in relation to the calculated values and as a result to their premature failures [4].

The usual control methods regarding coaxiality deviations do not fulfil the user's expectations in the case of gas turbine propulsion systems. The difficult access to flange connections, long control time, organisational difficulties and lack of qualified personnel creates the risk of incorrect measurements with errors exceeding allowable values. The aim of this paper was to describe the proposed vibroacoustic method that would replace the usual coaxiality control methods and would mitigate the operational hazards that were mentioned above.

The application of vibroacoustic diagnostics to technical maintenance makes it possible to lower the operational costs of the vessel by basing its operation on its actual technical condition and predicted failure states [1].

It was assumed that the determination of the relationship between the coaxiality parameters and the changes of the recorded vibration signals should result in identifying the proposed diagnostic model that consists of:

- The choice of geometrical parameters describing the position deviations, i.e. axis slope and displacement;

- The choice of adequate parameters of the vibroacoustic signal;

- The determination of mutual relationships between sets of the coaxiality deviations and vibration diagnostic parameter values;

- The sensitivity assessment of the symptoms in question;

- Establishing the database for statistical analysis and operational decision making.

The research in question was only limited to the control of the axis slope. This assumption was made to take into consideration the influence of the displacement hull deflection on the element position of the serial multi-shaft 
system. In this case the axis displacements are controlled solely during assembly of the shafting system in the production and repair stages.

The energy emitted as a result of a change of technical state of the flange connection was assumed to be reflected in the recorded vibration signal. As a result of the experiments conducted, the position and fitting direction of the accelerometers were chosen at the transverse cross-section of the transmission gear just over the main, radial bearing of the input shaft. On the basis of detail identification of the main signal against disturbances the lateral measurement direction with respect to the rotation axis was determined. The coaxiality measurements were carried out in the conditions of not transmitting the torque to the ship propeller.

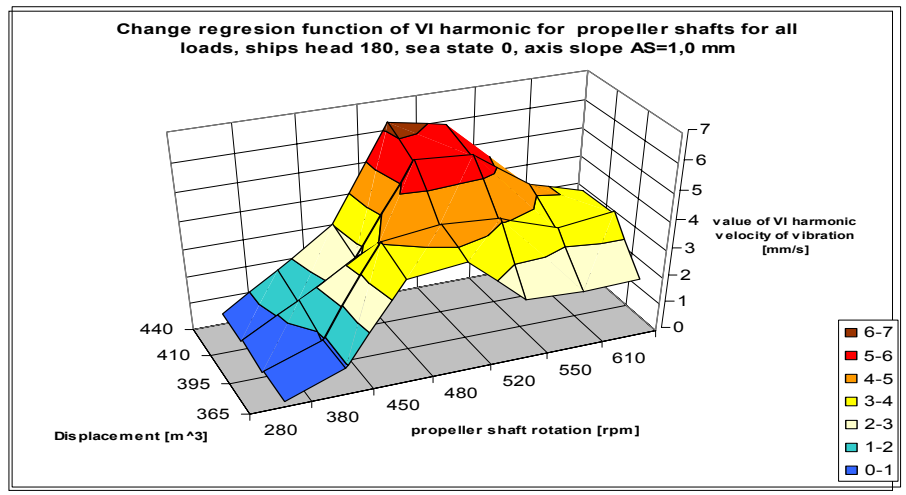

Figure 4: Exemplified archive charts of the investigation results.

The results were analysed by assigning the mean value of the vibration signal, i.e. the VI harmonic connected with the excitation frequency of vibration velocity amplitudes, to the measured axis slope value. Archive charts of the investigation results are demonstrated in Fig. 5. As a result of the analysis conducted, three conclusions with respect to general measurement principles could be drawn, as far as the investigated vessel is concerned:

1. It is important to determine the limiting values of the selected symptom at different vessel displacements. The proposed method can be applied in the entire range of the vessel's displacement.

2. The assessment of axis slope at the flexible coupling on the basis of the symptom in question is of secondary importance.

3. The selection of the accelerometer fitting position is the most important factor because of the close vicinity of the propulsion (reduction) gear main radial bearing. Accelerometer cantilevers applied during the measurements did not influence the measurement results, as their resonance frequencies were higher than that of the selected vibration symptom.

It was also stated, on the basis of analysis of the investigation results, that the mean signal value VI harmonics of the velocity vibration increases as the vessel's displacement increases, at the axis slope $Z=$ const. In order to recognize 
in a better manner the technical condition, the database is presented as $3 \mathrm{D}$ charts of different axils slope - as shown in Figure 4.

During the analysis conducted, no limit state of the axis slope of $Z=1 \mathrm{~mm} / \mathrm{m}$ was found in the propulsion system in question. Additionally, the regression function of the symptom changes at different vessel displacements was calculated in order to determine the limiting (tolerated) values of the symptom.

\section{Conclusions}

- The application of the proposed method results in an increased effectiveness of the management of the engine's operation time, especially at the end of the work cycle.

- The proposed approach is non-invasive and does not require that ships are put out of service.

- While performing the investigations as described in the paper, it is possible to collect data for a database for future monitoring system of ships. Such a database is expected to improve their operational features.

- Experience gained during the investigations could be utilised for other power plants equipped with gas turbines.

- The presented method possesses a degree of sensitivity that is sufficient for the purposes of the operation of the propulsion plant as it enables one to find the primary symptoms of changes to the technical state of rotordynamics.

\section{References}

[1] Charchalis A., Experimental diagnostics of naval gas turbines. ISROMAC'98 Honolulu (1998).

[2] Charchalis A., Diagnosing the ship shafting alignment within the ship power plant by means of vibration measurements. INTER NOISE'99, Fort Lauderdale (1999).

[3] Charchalis A., Multi Symptoms System of Diagnosing of Marine Gas Turbines. 3 International Congress of Technical Diagnostics, Poznan (2004)

[4] Charchalis A. Cwilewicz R. Grzadziela A. Diagnosing Elements of Propulsion Plant of Naval Vessels by Means of Vibration Measurement MECHANICS. 24 no.2. (2005)

[5] Charchalis A, Grządziela A, Diagnosing of naval gas turbine rotors with the use of vibroacoustic parameters. The 2001 International Congress and Exhibition on Noise Control Engineering. The Hague, The Netherlands (2001)

[6] Charchalis A., Diagnosing power plant and specialized equipment used on Poland's Navy Warship. II International Congress of Technical Diagnostics Warsaw (2000). 